\title{
Serum exosomal IncRNA SNHG7 is increased in NSCLC and predicts unfavorable prognosis
}

\section{Zhenkui Zhang ( $\square$ zhangzk_doctor84@126.com )}

Beijing Chao-Yang Hospital Capital Medical University: Beijing Chaoyang Hospital https://orcid.org/0000-0001-9214-3559

\section{Liqiang Zhao}

Beijing Chao-Yang Hospital Capital Medical University: Beijing Chaoyang Hospital

\section{Zhijian Wang}

Beijing Chao-Yang Hospital Capital Medical University: Beijing Chaoyang Hospital

\section{Lixin Yu}

Beijing Chao-Yang Hospital Capital Medical University: Beijing Chaoyang Hospital

\section{Fenghua Sun}

Beijing Chao-Yang Hospital Capital Medical University: Beijing Chaoyang Hospital

\section{Research}

Keywords: Non-small cell lung cancer, Exosome, SNHG7, Prognosis, Biomarker

Posted Date: October 18th, 2021

DOl: https://doi.org/10.21203/rs.3.rs-968553/v1

License: (c) (i) This work is licensed under a Creative Commons Attribution 4.0 International License.

Read Full License 


\section{Abstract}

\section{Background}

Exosomal long non-coding RNAs (IncRNAs) are proposed as promising non-invasive biomarkers for clinical applications.

\section{Aims}

In this study, we aimed to explore the potential of serum exosomal IncRNA SNHG7 for the diagnosis and prognosis prediction of non-small-cell lung cancer (NSCLC).

Methods

A total of 128 patients with NSCLC and 80 healthy volunteers were enrolled. Quantitative real-time polymerase chain reaction (qRT-PCR) was performed to measure the expression level of serum exosomal IncRNA SNHG7 in all participants. Receiver operating characteristic (ROC) analysis was used to analyze the diagnostic value of serum exosomal InCRNA SNHG7 and CEA for NSCLC. The relationship between serum exosomal IncRNA SNHG7 expression and clinical characteristics of NSCLC was also assessed.

Results

Serum exosomal IncRNA SNHG7 levels were significantly upregulated in NSCLC patients compared to controls. In addition, serum exosomal IncRNA SNHG7 yield a ROC area under the curve (AUC) value of 0.856 with $77.34 \%$ sensitivity and $83.75 \%$ specificity for identifying NSCLC patients from normal controls. Combining serum exosomal IncRNA SNHG7 and CEA improved the diagnostic accuracy of NSCLC, with an AUC of 0.932 with $88.28 \%$ sensitivity and $86.25 \%$ specificity. Moreover, serum exosomal SNHG7 levels were significantly downregulated in post-operative blood samples whereas markedly higher in patients who experienced a relapse. Significant associations of elevated serum exosomal IncRNA SNHG7 expression with TNM stage, lymphatic invasion and lymph node metastasis were observed. High serum exosomal SNHG7 expression was associated with poorer survival and served as an independent prognostic factor for NSCLC.

Conclusions

In conclusion, our data demonstrates that serum exosomal SNHG7 expression might be a potential valuable biomarker for NSCLC detection and prognosis prediction.

\section{Introduction}

In $2018,774,323$ new lung cancer cases and 690,567 new lung cancer deaths are projected to occur in China. Lung cancer accounts for $18 \%$ of all new diagnosed cancer and $24 \%$ of all cancer-related deaths [1]. Among all lung cancer cases, approximately $85 \%$ were non-small cell lung cancer (NSCLC) [2]. Despite 
the improvement of treatments in the past decades, the prognosis of this malignancy remains dismal. Unfortunately, most NSCLC cases are diagnosed at advanced clinical stage, the 5-year survival rates for this disease are unfavorable $[3,4]$. Thus, it is urgently needed to identify novel markers for the accurate diagnosis and prognosis prediction of NSCLC, which are important to improve the long-term survival rates.

Long non-coding RNAs (IncRNAs) is a type of non-coding RNAs of more than 200 nucleotides in length [5]. Many reports have revealed that IncRNAs are widely involved in the regulation of various biological processes, such as cell proliferation, invasion and metastasis [6, 7]. Dysregulation of IncRNAs has been found to be associated with the cancer progression, and IncRNAs might play as oncogenes or tumor suppressors in NSCLC. For instance, IncRNA PTAR upregulation significantly increased NSCLC cell proliferation, growth and functioned as an oncogene in NSCLC [8], while IncRNA LIFR-AS1 overexpression markedly restrained the carcinogenesis process and served as a tumor suppressor in NSCLC [9]. Exosomes are extracellular vesicles with a diameter about 40-100 nm and contain different molecules including IncRNAs. Recent studies have shown a number of exosomal IncRNAs are aberrantly expressed in NSCLC, such as 19H and DLX6-AS1 [10,11].

Small nucleolar RNA host gene 7 (SNHG7) is located on chromosome 9q34.3 and identified as an oncogene in NSCLC [12]. For example, IncRNA SNHG7 was confirmed to be significantly increased in NSCLC tissues and cells [13-15]. However, the potential clinical significance of serum exosomal IncRNA SNHG7 remains unknown. In this study, the levels of serum exosomal IncRNA SNHG7 were firstly detected, and the associations between serum exosomal SNHG7 expression and the prognosis of NSCLC were further analyzed.

\section{Materials And Methods}

\section{Study design and subjects}

The current study was approved by the Ethics Committee of Beijing Chao-yang Hospital, Capital Medical University, and each participant provided the signed written informed consent. A total of 128 patients with NSCLC and 80 healthy volunteers as controls were recruited. No patient had received any chemotherapy or radiotherapy before first time serum sample collection. The patients consisted of 102 males and 26 females with a medium age at 56.3 years old. Tumor stage was determined was according to the seventh edition of TNM staging system of the American Joint Committee on Cancer (AJCC). The demographic characteristics of all patients were summarized Table 1. All cases received regular follow-up. Overall survival (OS) time was calculated from the date of the initial surgery until the date of death or last followup. 
Table 1

Association of serum exosomal SNHG7 expression with clinical variables in NSCLC patients.

\begin{tabular}{|c|c|c|c|c|}
\hline Variables & $\begin{array}{l}\text { All } \\
\text { cases }\end{array}$ & $\begin{array}{l}\text { High serum exosomal } \\
\text { SNHG7 }\end{array}$ & $\begin{array}{l}\text { Low serum exosomal } \\
\text { SNHG7 }\end{array}$ & $P$-value \\
\hline Gender & & & & 0.3795 \\
\hline Male & 102 & 53 & 49 & \\
\hline Female & 26 & 11 & 15 & \\
\hline Age & & & & 0.1950 \\
\hline$<50$ years & 45 & 19 & 26 & \\
\hline$\geq 50$ years & 83 & 45 & 38 & \\
\hline Smoking status & & & & 0.2497 \\
\hline Never & 105 & 50 & 55 & \\
\hline Ever & 23 & 14 & 9 & \\
\hline Histological type & & & & 0.1063 \\
\hline Adenocarcinoma & 75 & 33 & 42 & \\
\hline $\begin{array}{l}\text { Squamous } \\
\text { carcinoma }\end{array}$ & 53 & 31 & 22 & \\
\hline Lymphatic invasion & & & & 0.0078 \\
\hline Negative & 59 & 22 & 37 & \\
\hline Positive & 69 & 42 & 27 & \\
\hline $\begin{array}{l}\text { Lymph node } \\
\text { metastasis }\end{array}$ & & & & 0.0002 \\
\hline Negative & 71 & 25 & 46 & \\
\hline Positive & 57 & 39 & 18 & \\
\hline TNM stage & & & & $<0.0001$ \\
\hline I/II & 72 & 23 & 49 & \\
\hline III/IV & 56 & 41 & 15 & \\
\hline
\end{tabular}

Peripheral blood samples were collected from all NSCLC patients and controls in EDTA-containing tubes. The samples were centrifuged at $1500 \mathrm{~g}$ for $10 \mathrm{~min}$ at $4^{\circ} \mathrm{C}$, and then stored at $-80^{\circ} \mathrm{C}$ until exosome isolation. In addition, post-operative blood samples were drawn from all NSCLC patients 30 days after surgery.

\section{Isolation of exosome}


Total exosomes were isolated using Total Exosome Isolation Reagent for Serum (Life Technologies, Austin, USA). Briefly, serum was thawed on ice and centrifuged at 2,000 $\mathrm{g}$ for $30 \mathrm{~min}$ to remove cells and debris. Next, ExoQuick Solution was added to serum samples, and the mixture was centrifuged at 1,500 $\mathrm{g}$ for $30 \mathrm{~min}$ after incubation. The supernatant was removed, followed by centrifugation at $1,500 \mathrm{~g}$ for $5 \mathrm{~min}$ to remove residual liquid. The exosome-containing pellets were resuspended in PBS and stored at $-80^{\circ} \mathrm{C}$ until RNA isolation.

$R N A$ extraction and quantitative real-time polymerase chain reaction ( $q R T-P C R)$

Total RNA in serum was isolated by using Trizol reagent (Invitrogen Corp, Carlsbad, CA, USA) following the manufacturer's protocol. NanoDrop ND-1000 spectrophotometer (Thermo Scientific, Wilmington, DE, USA) was used to detect the concentration of RNA. qRT-PCR was performed with SYBR Premix Ex Taq (Takara, Dalian, Liaoning, China) on the ABI PRISM 7900 Sequence Detector System (Applied Biosystems, CA, USA). Cel-miR-39 acted as a spike-in control for the expression of SNHG7, and the relative serum IncRNA SNHG7 expression was calculated using the $2^{-\triangle \Delta C t}$ method.

\section{Detection of CEA levels}

Carcinoma embryonic antigen (CEA) was a traditional tumor marker and currently widely used for NSCLC screening. In this study, electrochemiluminescent immunoassay (ECLIA, Hoffman-La Roche Ltd., Basel, Switzerland) was used to measure CEA levels according to the standard procedure.

\section{Statistical analysis}

All the data analyses were conducted using GraphPad Prism 8.2.1 software (GraphPad Software, Inc., USA). The Mann-Whitney $U$ test or Kruskal-Wallis test was performed to determine the significance in serum exosomal IncRNA SNHG7 expression. The correlation of serum exosomal IncRNA SNHG7 expression with clinical variables was analyzed by chi-square test. Receiver-operating characteristic (ROC) curves and area under the curve (AUC) were calculated to evaluate the clinical significance of serum exosomal IncRNA SNHG7 in NSCLC diagnosis. Kaplan-Meier methods and log-rank test was used to estimate OS of NSCLC patients. The significance of clinical variables was analyzed using multivariate Cox proportional hazards regression analysis. $P<0.05$ was considered statistically significant.

\section{Results}

Serum exosomal InCRNA SNHG7 expression was significantly higher NSCLC Serum exosomal IncRNA SNHG7 levels were compared between NSCLC patients and healthy volunteers. Figure $1 \mathrm{~A}$ showed that serum exosomal IncRNA SNHG7 levels were significantly higher in NSCLC patients $(P<0.0001)$. In addition, increased serum exosomal IncRNA SNHG7 levels were found in NSCLC patients in the advanced stage $(P<0.0001$, Figure $1 \mathrm{~B})$, or with positive lymphatic invasion $(P=0.0131$, Figure $1 \mathrm{C})$ or with positive lymph node metastasis $(P=0.0006$, Figure 1D). 
Diagnostic values of serum exosomal InCRNA SNHG7 and CEA in NSCLC patients ROC curve analysis demonstrated that serum exosomal IncRNA SNHG7 effectively discriminated NSCLC patients from normal controls with an AUC of 0.856 . The sensitivity was $77.34 \%$ and the specificity was $83.75 \%$, respectively (Figure 2A). Additionally, CEA had a sensitivity of $76.56 \%$ and a specificity of $82.50 \%$ for identifying NSCLC subjects from controls, with an AUC of 0.784 (Figure 2B). Combination of serum exosomal IncRNA SNHG7 expression and CEA revealed an increased AUC value of 0.932 , with a sensitivity of $88.28 \%$ and a specificity of $86.25 \%$ (Figure $2 \mathrm{C}$ ). Therefore, the combination of these two makers had better performance to differentiate NSCLC patients from healthy controls.

\section{Association of serum exosomal IncRNA SNHG7 with clinical variables in NSCLC patients}

To analyze the association between serum exosomal IncRNA SNHG7 levels and the clinical characteristics, all 128 NSCLC patients were divided into high expression group $(n=64)$ and low expression group $(n=64)$ based on the median serum exosomal IncRNA SNHG7 expression. As illustrated in Table 1, serum exosomal IncRNA SNHG7 was significantly associated with malignancy-related factors, including positive lymphatic invasion $(P=0.0078)$, positive lymph node metastasis $(P=0.0002)$ and advanced TNM stage $(P<0.0001)$. However, the differences were not statistically significant between serum exosomal IncRNA SNHG7 expression and gender $(P=0.3795)$, age $(P=0.1950)$, smoking status $(P=0.2497)$, and histological type $(P=0.1063)$.

\section{Serum exosomal IncRNA SNHG7 levels before and after surgery}

As presented in Figure 3A, serum exosomal IncRNA SNHG7 expression levels in the post-operative blood samples were markedly reduced following surgery $(P<0.0001)$. During the follow-up, 12 patients relapsed $(12 / 64,18.8 \%)$ among patients with low serum exosomal IncRNA SNHG7 expression, while 46 patients relapsed $(46 / 64,71.9 \%)$ among patients with high serum exosomal IncRNA SNHG7 expression. Blood samples were obtained from 58 patients with disease relapse, and serum exosomal IncRNA SNHG7 expression was detected with qRT-PCR. Serum exosomal IncRNA SNHG7 levels in the relapse samples $(\mathrm{n}=58)$ were greatly higher than in the pre-operative blood samples $(P<0.0001$, Figure 3B).

\section{Association between serum exosomal IncRNA SNHG7 levels and OS}

Kaplan-Meier analysis revealed that NSCLC patients with higher levels of serum exosomal IncRNA SNHG7 had significantly shorter OS in comparison with those with lower expression levels $(P=0.0128$, Figure 4A). Moreover, 58 relapsed patients suffered a significantly worse survival rate $(P<0.0001$, Figure 4B). In the multivariate analysis, serum exosomal IncRNA SNHG7 ( $\mathrm{HR}=4.35,95 \% \mathrm{Cl}=2.36-6.89, P=0.009)$, lymphatic invasion $(\mathrm{HR}=3.58,95 \% \mathrm{Cl}=1.92-5.46, P=0.026)$, lymph node metastasis $(\mathrm{HR}=4.13,95 \%$ 
$\mathrm{Cl}=2.21-6.58, P=0.011)$ and TNM stage $(\mathrm{HR}=4.97,95 \% \mathrm{Cl}=2.66-7.53, P<0.001)$ were independent prognostic indicators for OS in NSCLC (Table 2).

Table 2

Multivariate analysis of overall survival in 128 NSCLC patients.

\begin{tabular}{|llll|}
\hline Parameters & Hazard Ratio & $95 \% \mathrm{Cl}$ & P-value \\
\hline Gender & 1.23 & $0.98-1.62$ & 0.104 \\
\hline Age & 2.13 & $1.31-2.97$ & 0.076 \\
\hline Smoking status & 1.56 & $1.15-2.14$ & 0.093 \\
\hline Histological type & 2.42 & $1.48-3.62$ & 0.065 \\
\hline Lymphatic invasion & 3.58 & $1.92-5.46$ & 0.026 \\
\hline Lymph node metastasis & 4.13 & $2.21-6.58$ & 0.011 \\
\hline TNM stage & 4.97 & $2.66-7.53$ & $<0.001$ \\
\hline Serum exosomal SNHG7 & 4.35 & $2.36-6.89$ & 0.009 \\
\hline
\end{tabular}

\section{Discussion}

Nowadays, clinical scientists still face the challenge of improving the early diagnosis and prognosis prediction of NSCLC. Some tumor markers, such as CEA, have low specificity and sensitivity for the early detection of this disease. Thus, exploring novel biomarkers for NSCLC are urgently required. In this study, we showed that serum exosomal SNHG7 expression was significantly higher in NSCLC patients. High serum exosomal SNHG7 expression occurred more frequently in NSCLC patients with advanced TNM stage, positive lymphatic invasion and positive lymph node metastasis. In addition, serum exosomal SNHG7 showed high accuracy in differentiating NSCLC patients from normal controls, and a combination of serum exosomal SNHG7 and CEA could yield improved the diagnosis of NSCLC. Moreover, increased serum exosomal SNHG7 expression was strongly associated with aggressive clinical parameters.

Furthermore, serum exosomal SNHG7 levels were remarkably decreased after surgery, and patients with relapse exhibited significantly higher serum exosomal SNHG7 levels. Finally, NSCLC patients with high serum exosomal SNHG7 expression had worse survival, and serum exosomal SNHG7 was considered as an independent prognostic indicator for OS.

Our findings were consistent with previously reported results. For instance, Pang et al showed SNHG7 was significantly overexpressed in NSCLC tissues and cells. Downregulation of SNHG7 greatly inhibited cell proliferation, metastasis and epithelial to mesenchymal transition (EMT) in vitro and carcinogenesis in vivo [13]. Likewise, SNHG7 was involved in the NSCLC progression by sponging miR-181a-5p. In vitro and in vivo analysis showed that SNHG7 inhibition or miR-181a-5p upregulation markedly decreased cell proliferation, stimulated cell apoptosis and attenuated tumor growth, and vice versa $[14,15]$. The data showed SNHG7 might serve as an oncogene in NSCLC. 
Accumulating evidence have also shown the oncogenic activities of SNHG7 in different types of cancer. In hepatocellular carcinoma (HCC), SNHG7 expression levels were remarkably upregulated in HCC tissues in comparison with adjacent normal tissues. SNHG7 overexpression was strongly associated aggressive clinical parameters and worse prognosis of HCC patients, and SNHG7 knockdown significantly decreased cancer cell proliferation, migration and invasion by inversely regulating RBM5 and RPL4 expression [1618]. In colorectal cancer (CRC), SNHG7 overexpression was found both in CRC tissues and cell lines. SNHG7 upregulation significantly stimulated cell proliferation, metastasis in vitro and promoted tumorigenesis in vivo $[19,20]$. In addition, SNHG7 expression was remarkably increased in cervical cancer (CC) tissues and cell lines. SNHG7 inhibition significantly attenuated cancer cell proliferation, invasion, migration and induced cell apoptosis through miR-485-5p/JUND axis. CC patients with higher IncRNA SNHG7 expression had shorter survival time [21, 22]. Moreover, SNHG7 expression was upregulated in breast cancer (BC) tissues and cells. High SNHG7 expression was closely associated with tumor stage, lymph node metastasis and worse overall survival of BC patients. SNHG7 inhibition enhanced the sensitivity of $\mathrm{BC}$ cells to trastuzumab, reduced cell proliferative, invasive abilities and promoted cell apoptosis by upregulating miR-186 [23, 24]. In gastric cancer (GC), high expression of SNHG7 occurred more frequently in in cancerous tissues and cell lines. Increased SNHG7 expression was highly associated with advanced clinical stage, lymph node metastasis and distant metastasis. In vitro and in vivo evidence demonstrated that SNHG7 suppression markedly inhibited the cancer progression of GC $[25,26]$.

\section{Conclusion}

In conclusion, we have demonstrated that serum exosomal SNHG7 expression is dramatically higher in NSCLC, and its upregulation is strongly associated with unfavorable prognosis of NSCLC. Therefore, serum exosomal SNHG7 might serve as a non-invasive and robust biomarker for NSCLC diagnosis and prognosis prediction.

\section{Declarations}

\section{Acknowledgements}

All authors have agreed to all of the contents and have approved the manuscript for submission. The content of the manuscript has not been published or submitted for publication elsewhere.

\section{Funding}

not applicable

\section{Ethics declarations}

\section{Ethics approval and consent to participate}


The current study was approved by the Ethics Committee of Beijing Chao-yang Hospital, Capital Medical University (approval no. 20181172). Each participant provided the signed written informed consent.

\section{Consent for publication}

All authors read and approved the final manuscript.

\section{Competing interests}

The authors declare no conflict of interest.

\section{References}

1. Rui-Mei Feng, Yi-Nan Zong, Su-Mei Cao, Rui-Hua Xu. Current cancer situation in China: good or bad news from the 2018 Global Cancer Statistics? Cancer Commun (Lond). 2019; 39(1): 22.

2. Pei Luo, Qing Yang, Le-Le Cong, Xiao-Feng Wang, Yu-Sheng Li, Xiao-Ming Zhong, Ru-Ting Xie, ChengYou Jia, Hui-Qiong Yang, Wen-Ping Li, Xian-Ling Cong, Qing Xia, Da Fu, Qing-Hua Zeng, Yu-Shui Ma. Identification of miR-124a as a novel diagnostic and prognostic biomarker in non-small cell lung cancer for chemotherapy. Mol Med Rep. 2017; 16(1): 238-246.

3. Siegel, RL, Miller, KD, Jemal, A. Cancer statistics, 2015. CA Cancer J Clin 2015; 65(1): 5-29.

4. Li, XX, Li, RJ, Zhao, LJ. Expression of molecular factors correlated with metastasis in small cell lung cancer and their significance. Int J Clin Exp Pathol 2015; 8(11): 14676-14684.

5. Adam M Schmitt, Howard Y Chang. Long noncoding RNAs in cancer pathways. Cancer Cell. 2016; 29(4): 452-463.

6. Jinglin Li, Zhenglong Li, Wangyang Zheng, Xinheng Li, Zhidong Wang, Yunfu Cui, Xingming Jiang. LncRNA-ATB: An indispensable cancer-related long noncoding RNA. Cell Prolif. 2017; 50(6): e12381.

7. Batista PJ, Chang HY. Long noncoding RNAs: cellular address codes in development and disease. Cell. 2013; 152: 1298-1307.

8. Wenjun Yu, Zhenni Sun, Ling Yang, Yafei Han, Lu Yue, Lihua Deng, Ruyong Yao. LncRNA PTAR promotes NSCLC cell proliferation, migration and invasion by sponging microRNA-101. Mol Med Rep. 2019; 20(5): 4168-4174.

9. Qun Wang, Jing Wu, Hui Huang, Yan Jiang, Ying Huang, Hongyan Fang, Gang Zheng, Xiaochun Zhou, Yujuan Wu, Changjiang Lei, Desheng Hu. LncRNA LIFR-AS1 suppresses invasion and metastasis of nonsmall cell lung cancer via the miR-942-5p/ZNF471 axis. Cancer Cell Int. 2020; 20: 180.

10. Tianyi Gao, Xiangxiang Liu, Bangshun He, Zhenlin Nie, Chengbin Zhu, Pei Zhang, Shukui Wang. Exosomal IncRNA $91 \mathrm{H}$ is associated with poor development in colorectal cancer by modifying HNRNPK 
expression. Cancer Cell Int. 2018; 18: 11.

11. Xilin Zhang, Huihui Guo, Ying Bao, Huanming Yu, Dong Xie, Xiang Wang. Exosomal long non-coding RNA DLX6-AS1 as a potential diagnostic biomarker for non-small cell lung cancer. Oncol Lett. 2019; 18(5): 5197-5204.

12. Chaudhry MA. Expression pattern of small nucleolar RNA host genes and long non-coding RNA in Xrays-treated lymphoblastoid cells. Int J Mol Sci. 2013; 14(5): 9099-9110.

13. Lingling Pang, Yun Cheng, Shenchun Zou, Jie Song. Long noncoding RNA SNHG7 contributes to cell proliferation, migration, invasion and epithelial to mesenchymal transition in non-small cell lung cancer by regulating miR-449a/TGIF2 axis. Thorac Cancer 2020; 11(2): 264-276.

14. Liming Wang, Lili Zhang, Liwei Wang. SNHG7 contributes to the progression of non-small-cell lung cancer via the SNHG7/miR-181a-5p/E2F7 axis. Cancer Manag Res. 2020; 12: 3211-3222.

15. Liping Li, Dan Ye, Liang Liu, Xiaoju Li, Jun Liu, Shengtian Su, Wenjing Lu, Zhigao Yu. Long noncoding RNA SNHG7 accelerates proliferation, migration and invasion of non-small cell lung cancer cells by suppressing miR-181a-5p through AKT/mTOR signaling pathway. Cancer Manag Res. 2020; 12: 83038312.

16. An Shen, Jinping Ma, Xiaolin Hu, Xinjiang Cui. High expression of IncRNA-SNHG7 is associated with poor prognosis in hepatocellular carcinoma. Oncol Lett. 2020; 19(6): 3959-3963.

17. B-Z Sun, D-G Ji, Z-X Feng, Y Wang. Long noncoding RNA SNHG7 represses the expression of RBM5 to strengthen metastasis of hepatocellular carcinoma. Eur Rev Med Pharmacol Sci. 2019; 23(13): 56995704.

18. Xue Yang, Liankang Sun, Liang Wang, Bowen Yao, Huanye Mo, Wei Yang. LncRNA SNHG7 accelerates the proliferation, migration and invasion of hepatocellular carcinoma cells via regulating miR-122-5p and RPL4. Biomed Pharmacother. 2019; 118: 109386.

19. Yujia Shan, Jia Ma, Yue Pan, Jialei Hu, Bing Liu, Li Jia. LncRNA SNHG7 sponges miR-216b to promote proliferation and liver metastasis of colorectal cancer through upregulating GALNT1. Cell Death Dis. 2018; 9(7): 722.

20. Yang Li, Changqian Zeng, Jialei Hu, Yue Pan, Yujia Shan, Bing Liu, Li Jia. Long non-coding RNASNHG7 acts as a target of miR-34a to increase GALNT7 level and regulate PI3K/Akt/mTOR pathway in colorectal cancer progression. J Hematol Oncol. 2018; 11(1): 89.

21. Danyang Zhao, Hui Zhang, Jianxiong Long, Mujun Li. LncRNA SNHG7 functions as an oncogene in cervical cancer by sponging miR-485-5p to modulate JUND expression. Onco Targets Ther. 2020; 13 : 1677-1689. 
22. J Zeng, Y-X Ma, Z-H Liu, Y-L Zeng. LncRNA SNHG7 contributes to cell proliferation, invasion and prognosis of cervical cancer. Eur Rev Med Pharmacol Sci. 2019; 23(21): 9277-9285.

23. X Luo, Y Song, L Tang, D-H Sun, D-G Ji. LncRNA SNHG7 promotes development of breast cancer by regulating microRNA-186. Eur Rev Med Pharmacol Sci. 2018; 22(22): 7788-7797.

24. Hui Zhang, Xiao-Yu Zhang, Xiao-Ning Kang, Li-Jun Jin, Zun-Yi Wang. LncRNA-SNHG7 enhances chemotherapy resistance and cell viability of breast cancer cells by regulating miR-186. Cancer Manag Res. 2020; 12: 10163-10172.

25. M-W Wang, J Liu, Q Liu, Q-H Xu, T-F Li, S Jin, T-S Xia. LncRNA SNHG7 promotes the proliferation and inhibits apoptosis of gastric cancer cells by repressing the P15 and P16 expression. Eur Rev Med Pharmacol Sci. 2017; 21(20): 4613-4622.

26. Yangmei Zhang, Yuan Yuan, Youwei Zhang, Long Cheng, Xichang Zhou, Kai Chen. SNHG7 accelerates cell migration and invasion through regulating miR-34a-Snail-EMT axis in gastric cancer. Cell Cycle. 2020; 19(1): 142-152.

\section{Figures}


A

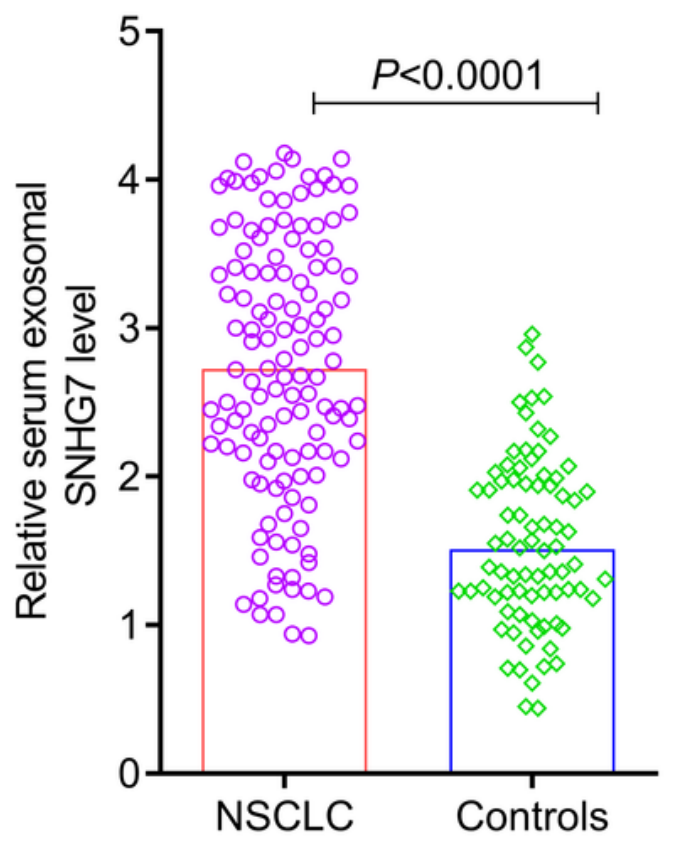

C

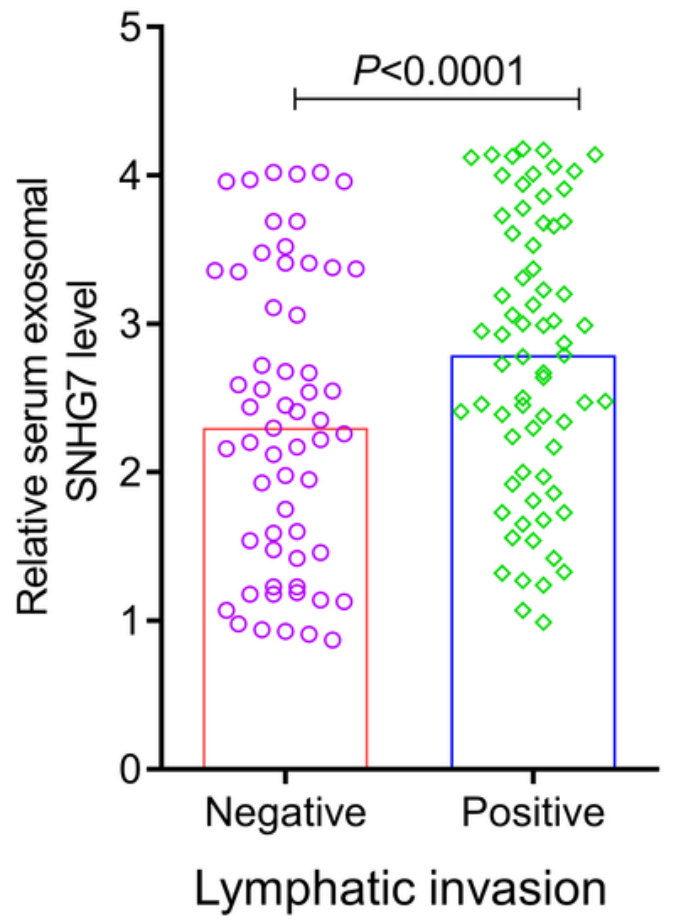

B

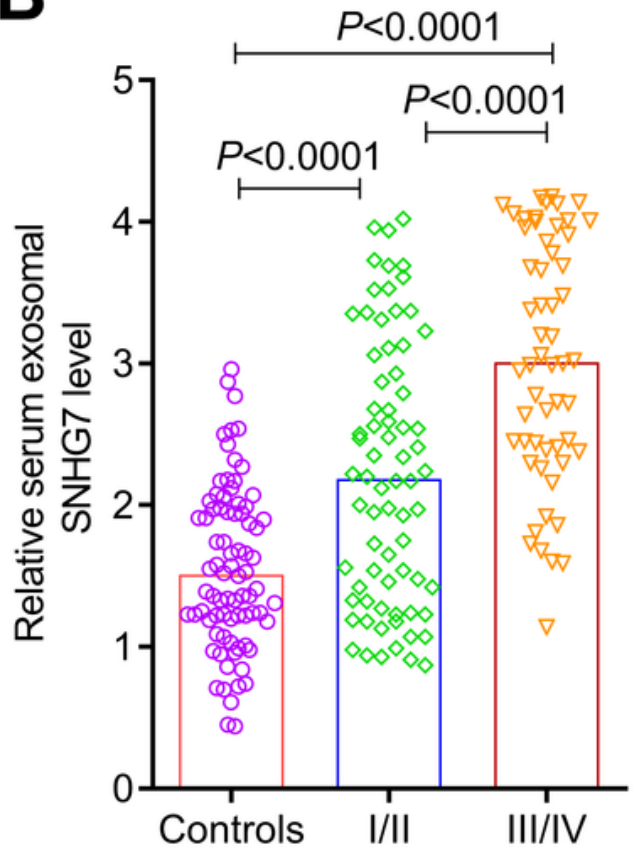

D

TNM stage

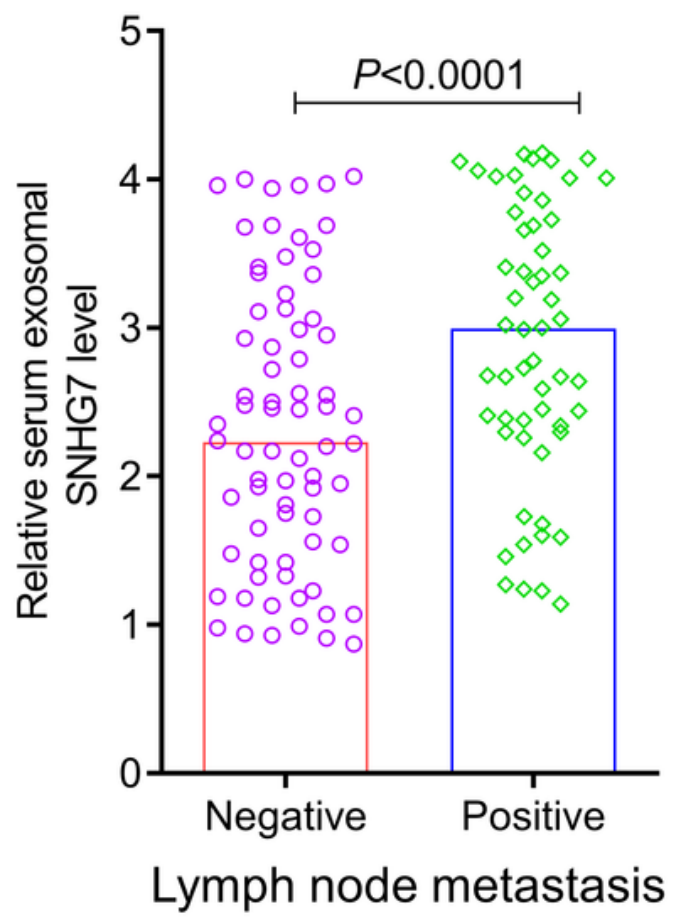

\section{Figure 1}

Serum exosomal IncRNA SNHG7 was increased in NSLCC. (A) Serum exosomal IncRNA SNHG7 levels in 128 NSCLC patients and 80 controls. (B) Serum exosomal IncRNA SNHG7 levels in controls and NSCLC patients at different TNM stages. (C) Serum exosomal IncRNA SNHG7 levels in NSCLC patients with or without lymphatic invasion. (D) Serum exosomal IncRNA SNHG7 levels in NSCLC patients with or without lymph node metastasis 
A

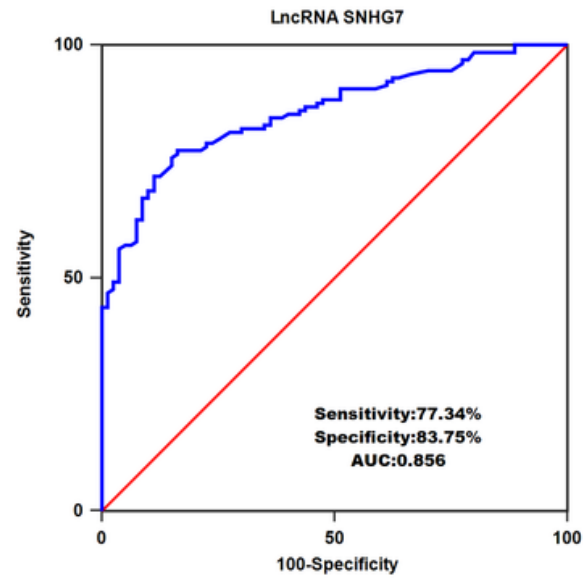

B

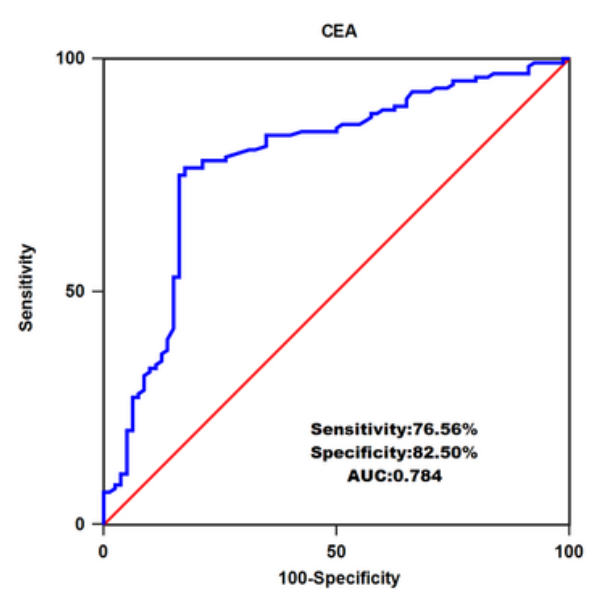

C

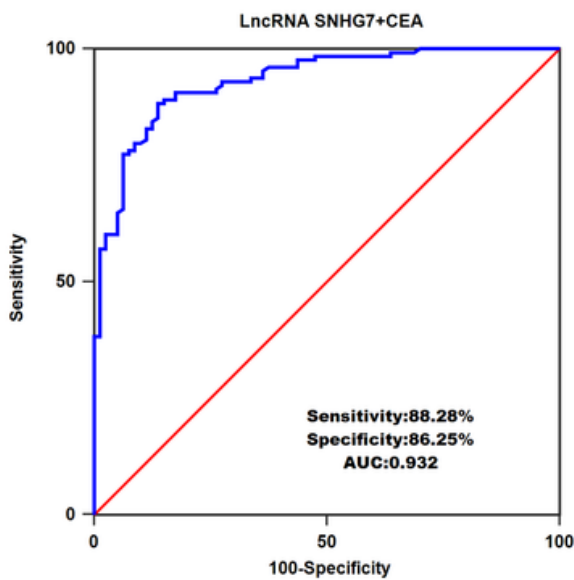

Figure 2

ROC curve analysis for serum exosomal IncRNA SNHG7 (A), CEA (B) and the combination of two markers in the diagnosis of NSCLC.

A

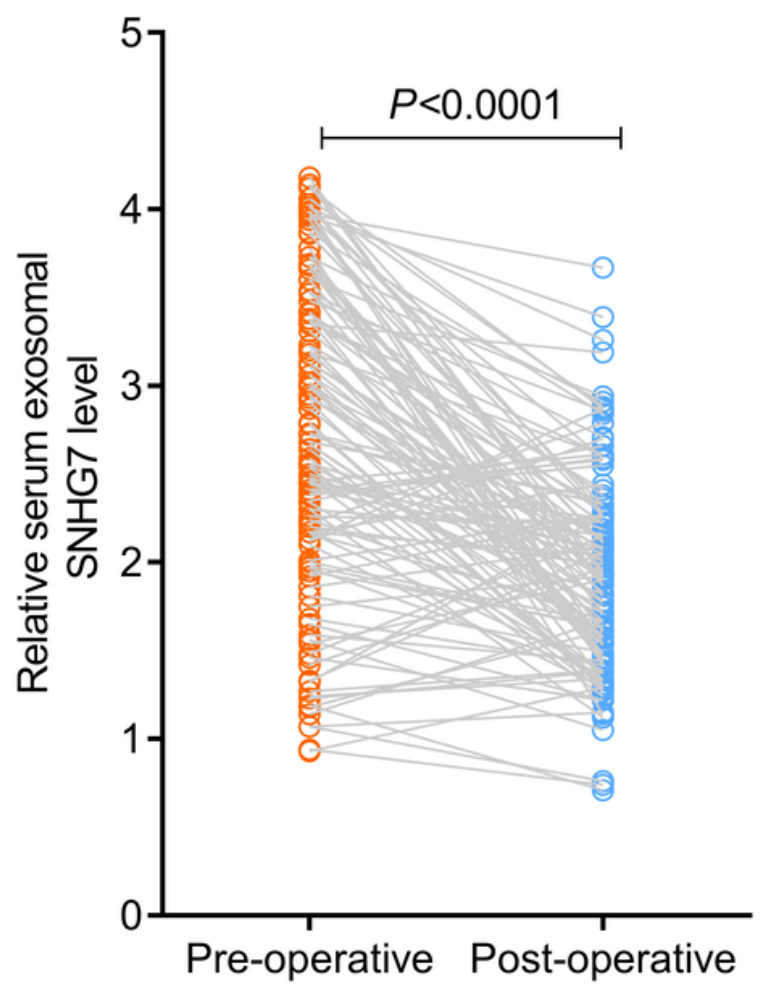

B

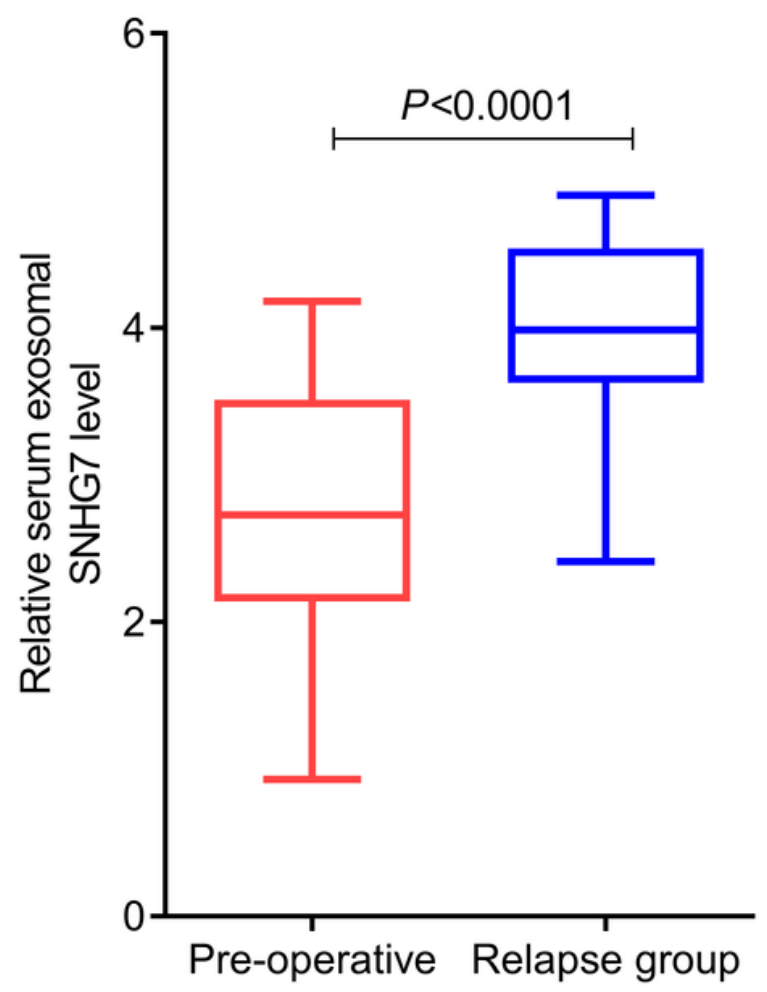

\section{Figure 3}

(A) Comparison of serum exosomal IncRNA SNHG7 levels between preoperative and postoperative samples. (B) Patients in the relapse group had significantly higher serum exosomal IncRNA SNHG7 levels 
than those in pre-operative blood sample of all NSCLC patients.

A

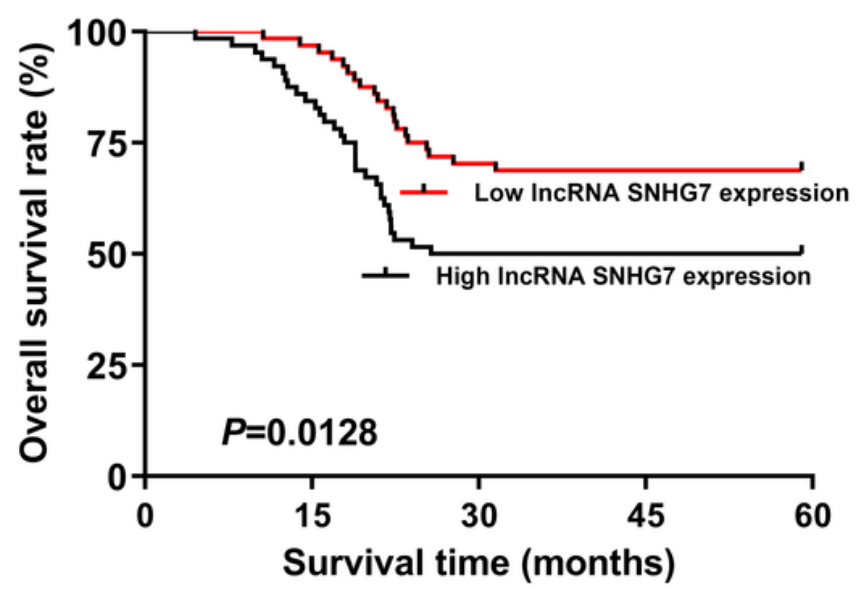

B

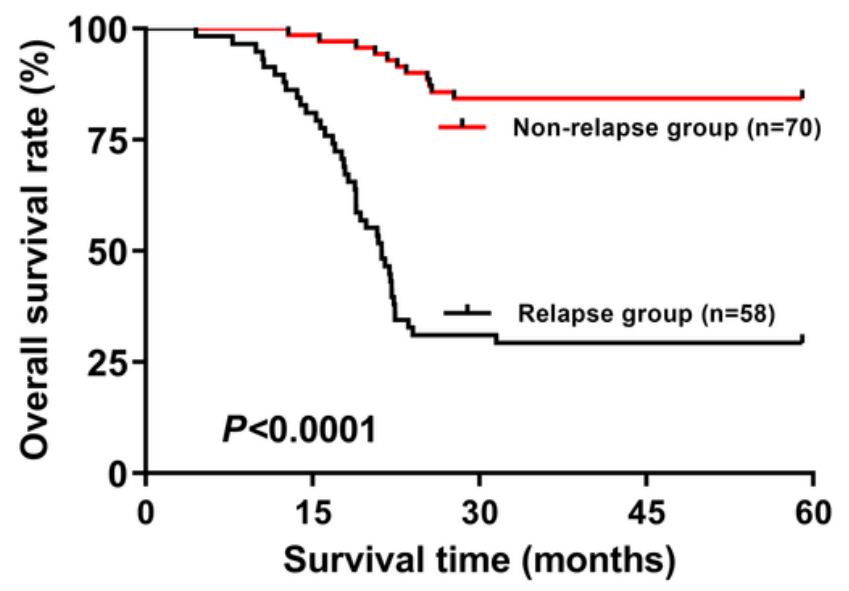

\section{Figure 4}

(A) For all the NSCLC cases, patients with higher exosomal IncRNA SNHG7 had worse survival. (B) Compared to the patients in the relapse group, patients in the non-relapse group had better survival. 